\title{
Electronic Coupling between Two Covalently Bonded Dimolybdenum Units Bridged by A Naphthalene Group
}

Guang Yuan Zhu, $\uparrow$ Miao Meng, $\uparrow$ Ying Ning Tan, Xuan Xiao and Chun Y. Liu*

Department of Chemistry, Jinan University, 601 Huang-Pu Avenue West, Guanzhou 510632, China.

Supporting Information

- Syntheses of the two thiolated bridging ligands

- ${ }^{1}$ H NMR spectra

- DPV of the dimeric complexes

- Electronic spectra of the dimeric complexes

- EPR spectra of the mixed-valence complexes

- Crystallographic data and structural parameters

- DFT calculation data 
- Syntheses of the two thiolated bridging ligands
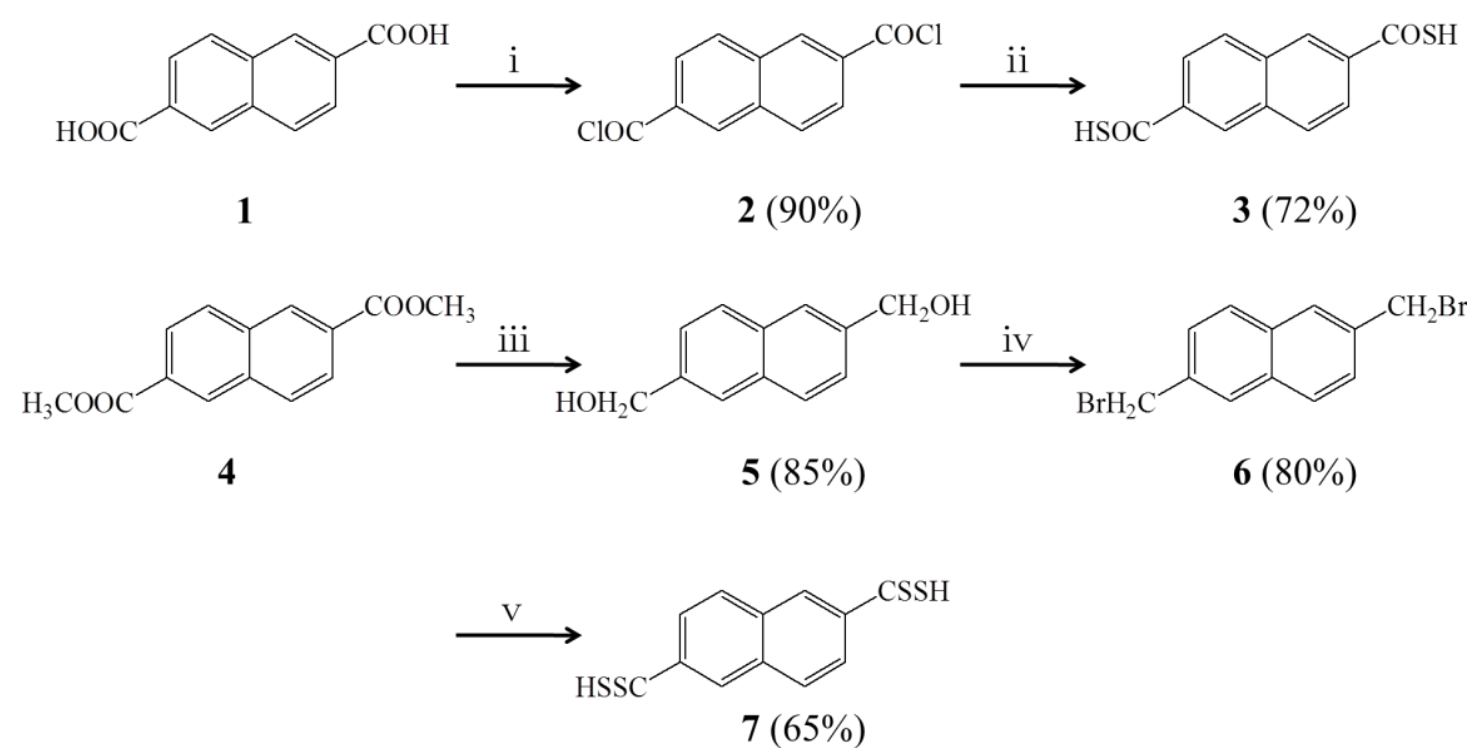

Scheme S1. (i) $\mathrm{SOCl}_{2}$, DMF, $90{ }^{\circ} \mathrm{C}$ reflux, 3 h. (ii) $\mathrm{CH}_{3} \mathrm{CSNH}_{2}$, THF, r.t., 3 h; $\mathrm{NaOH}$, $\mathrm{HCl}$; (iii) $\mathrm{LiAlH}_{4}$, THF, $0{ }^{\circ} \mathrm{C}, 0.5 \mathrm{~h}$; $70{ }^{\circ} \mathrm{C}$ reflux, $24 \mathrm{~h}$; (iv) $\mathrm{PBr}_{3}, \mathrm{DMF} / \mathrm{DCM}$ (v:v= 1/3), r.t., 2 h; $\mathrm{H}_{2} \mathrm{O}$; (v) S, $\mathrm{NaOCH}_{3}, \mathrm{CH}_{3} \mathrm{OH}, 90^{\circ} \mathrm{C}$ reflux, 12 h; $\mathrm{HCl}$.

Preparation of 2,6-dithionaphthalenedicarboxylic acid (3). A mixture of 2,6-naphthalenedicarboxylic acid $(0.43 \mathrm{~g}, 2.00 \mathrm{mmol})$ and thionyl chloride $(15 \mathrm{~mL})$ in the presence of catalytic amount of DMF was refluxed at $90{ }^{\circ} \mathrm{C}$ for $12 \mathrm{~h}$. The resltant light-green solution was cooled to room temperature and excess thionyl chloride was removed under reduced pressure. The residue was washed with hexane $(3 \times 15 \mathrm{~mL})$ and then dried by vacuum. Yield: $0.45 \mathrm{~g}(90 \%)$.This material (2) was used for preparation of $\mathbf{3}$ without further purification.

A mixture of the crude product of $2(0.25 \mathrm{~g}, 1.00 \mathrm{mmol})$ and thioacetamide $(0.20$ $\mathrm{g}, 2.50 \mathrm{mmol})$ in THF $(20 \mathrm{~mL})$ was stirred at room temperature for $3 \mathrm{~h}$.Then $\mathrm{NaOH}(1$ M, $20 \mathrm{~mL}$ ) was carefully added to the reaction mixture. The solvents were evaporated under reduced pressure, and then dilute hydrochloric acid (1 M, $30 \mathrm{~mL})$ was added. The water layer was extracted with diethyl ether $(3 \times 30 \mathrm{~mL})$. The organic layer (light-yellow) was evaporated by rotavapor, producing a yellow solid. The product was collected and dried under vacuum. Yield: $0.20 \mathrm{~g}(72 \%) .{ }^{1} \mathrm{H}$ NMR $\delta$ (ppm in DMSO): $8.07(\mathrm{~d}, 2 \mathrm{H}$, aromatic $\mathrm{C}-H), 8.22(\mathrm{~d}, 2 \mathrm{H}$, aromatic $\mathrm{C}-H), 8.68(\mathrm{~s}, 2 \mathrm{H}$, 
aromatic $\mathrm{C}-H)$.

Preparation of 2,6-naphthalenetetrathiodicarboxylic acid (7). In the ice water bath, $\mathrm{LiAlH}_{4}(0.76 \mathrm{~g}, 0.02 \mathrm{~mol})$ suspended in $30 \mathrm{~mL}$ of THF was transferred slowly to a solution of 2,6-dimethylnaphthalenecarboxylate (4) $(2.44 \mathrm{~g}, 0.01 \mathrm{~mol})$ in $50 \mathrm{~mL}$ of THF. The mixture was stirred for $30 \mathrm{~min}$ at low temperature and then at room temperature for another $30 \mathrm{~min}$. The reaction was refluxed at $70{ }^{\circ} \mathrm{C}$ for $24 \mathrm{~h}$. The mixture was cooled to room temperature and the excess $\mathrm{LiAlH}_{4}$ was quenched by methanol. After removal of the solvents under reduced pressure, dilute hydrochloric acid $(1 \mathrm{M}, 30 \mathrm{~mL})$ and ethyl acetate were added to extract the product. The organic layer (light-yellow) was separated. Evaporation of the solvent gave a yellow solid. Yield: $1.88 \mathrm{~g}(85 \%)$. This material (5) was used for preparation of $\mathbf{6}$ without purification.

In ice water bath, $\mathrm{PBr}_{3}(1.35 \mathrm{~g}, 5 \mathrm{mmol})$ was transferred dropwise to a solution of $5(0.94 \mathrm{~g}, 5 \mathrm{mmol})$ in $20 \mathrm{~mL}$ DCM mixed with DMF (v:v = 3:1). After $30 \mathrm{~min}$ stirring, water of $20 \mathrm{~mL}$ was added. The aqueous layer was extracted with DCM $(3 \times$ $20 \mathrm{~mL}$ ). The organic layer was evaporated by rotavapor, producing a light-yellow solid. This crude product was washed with diethyl ether $(3 \times 15 \mathrm{~mL})$ and collected by filtration. The product was dried under vacuum. Yield: $1.25 \mathrm{~g}(80 \%)$. This material (6) was used for preparation of 7 without purification.

A solution of sodium methoxide $(0.43 \mathrm{~g}, 8 \mathrm{mmol})$ in $10 \mathrm{~mL}$ of methanol was transferred to a flask with sulfur $(0.26 \mathrm{~g}, 8 \mathrm{mmol})$ suspending in $30 \mathrm{~mL}$ of methanol. The mixture was refluxed at $70{ }^{\circ} \mathrm{C}$ for $2 \mathrm{~h}$. After cooled to room temperature, a solution of $6(0.63 \mathrm{~g}, 2 \mathrm{mmol})$ in $30 \mathrm{~mL}$ methanol was transferred. then refluxed at 70 ${ }^{\circ} \mathrm{C}$ for another $7 \mathrm{~h}$. The solvent of methanol was evaporated under reduced pressure, and then dilute hydrochloric acid $(1 \mathrm{M}, 30 \mathrm{~mL})$ was added. The water layer was extracted with DCM $(3 \times 30 \mathrm{~mL})$. The organic layer $(\mathrm{red})$ was evaporated by rotavapor, producing a red solid (7). Yield: $0.36 \mathrm{~g}(65 \%)$ 
- ${ }^{1} H$ NMR spectra of the neutral complexes

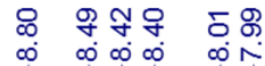

กิ

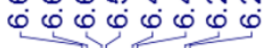

న⿻्ष

1

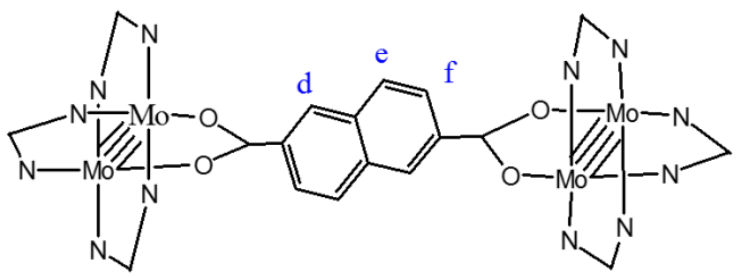<smiles>C=NCN=CNc1ccc(OC)cc1</smiles>

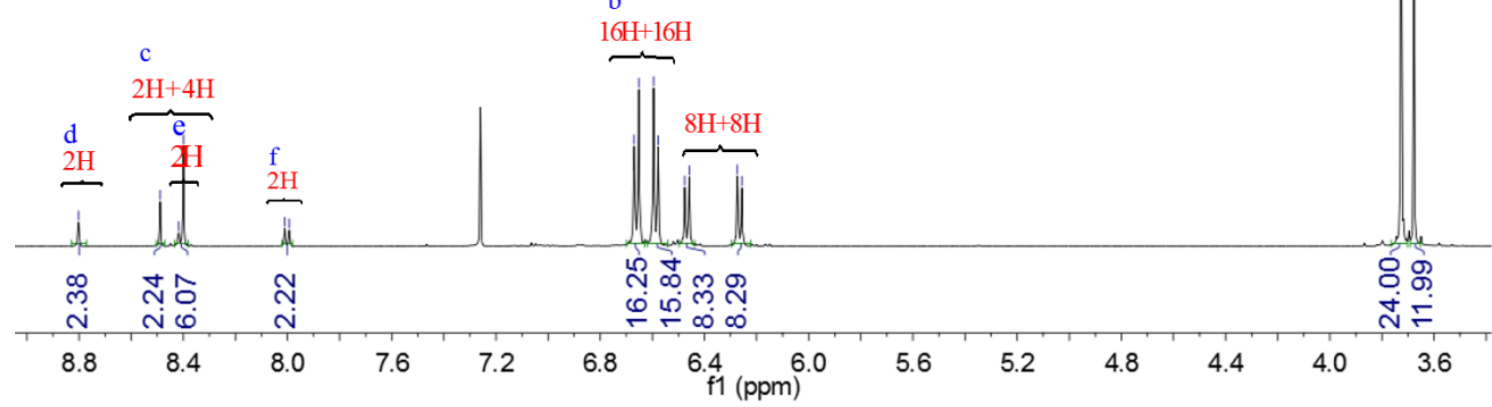

Figure S1. ${ }^{1} \mathrm{H}$ NMR spectrum for $\left[\mathbf{O}_{2}-(\mathbf{2}, \mathbf{6}-\mathbf{n a p h})-\mathbf{O}_{\mathbf{2}}\right]$.

\section{웅요용 \&}

$\infty$

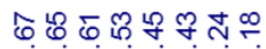

ம

NN\&
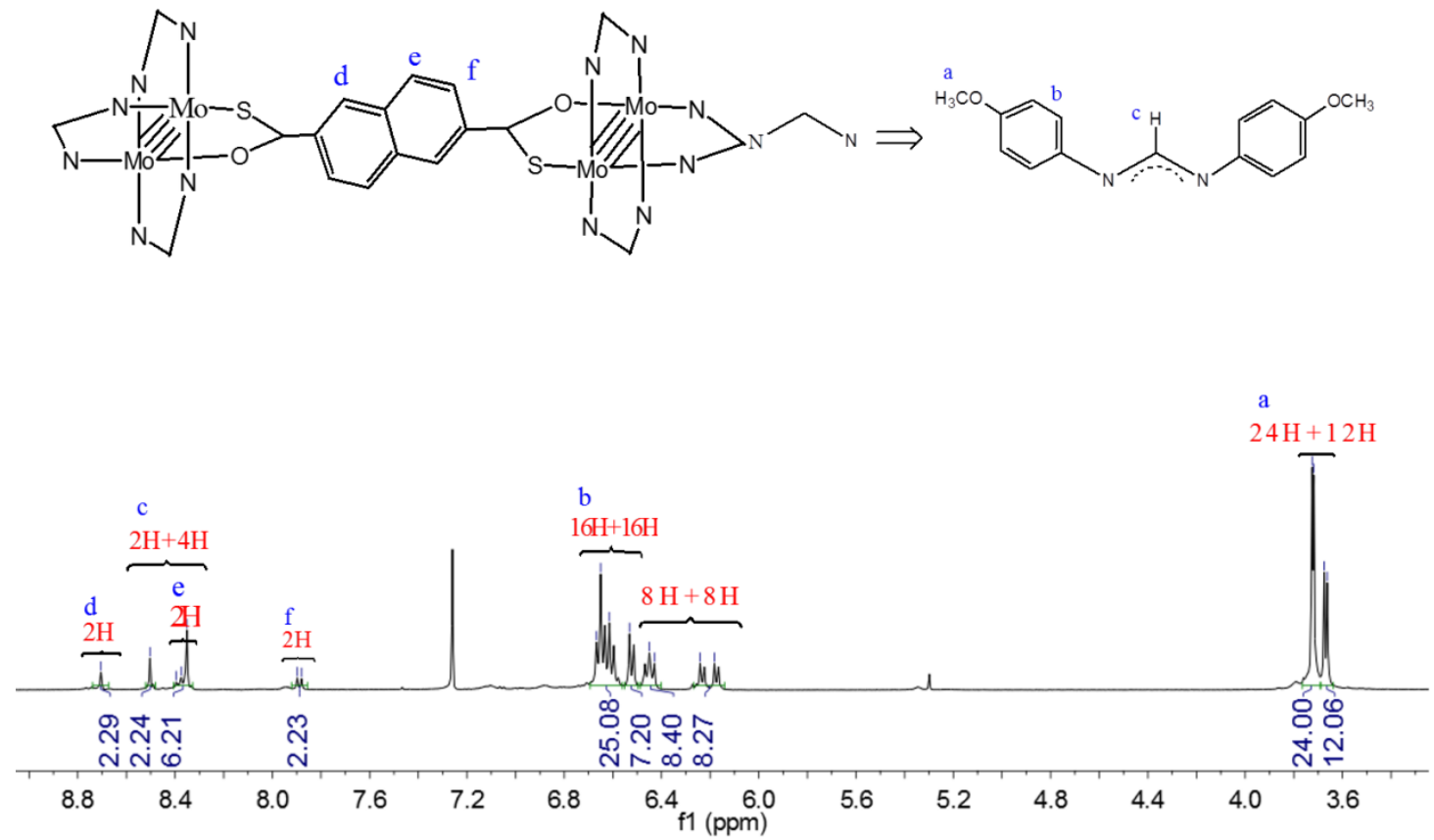

Figure S2. ${ }^{1} \mathrm{H}$ NMR spectrum for [OS-(2,6-naph)-OS] 


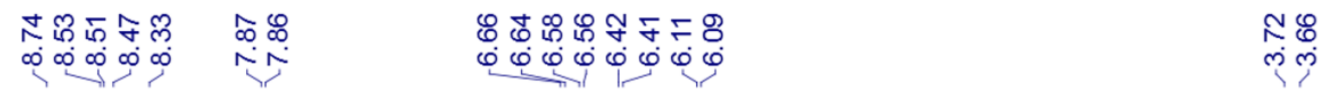
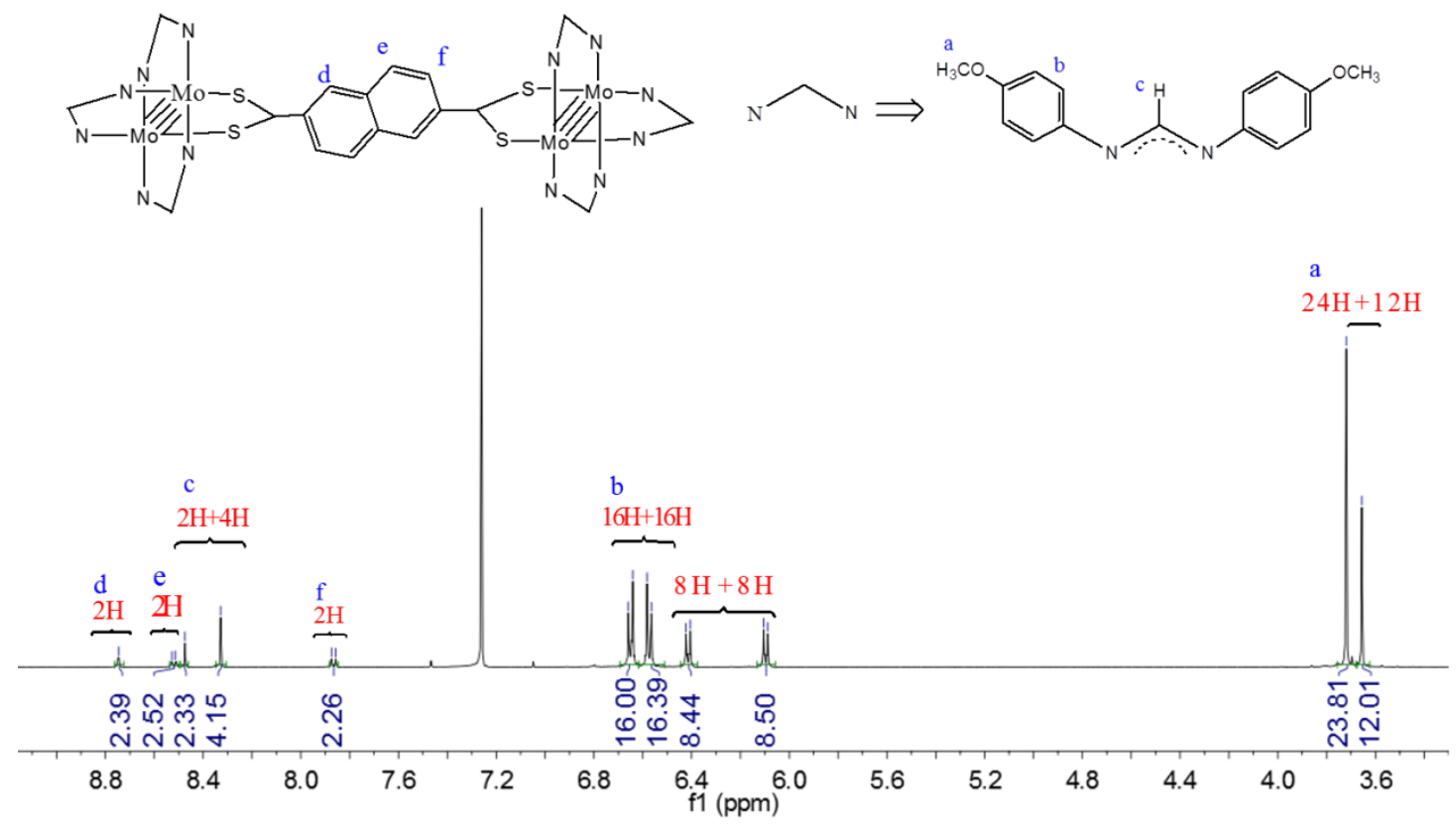

Figure S3. ${ }^{1} \mathrm{H}$ NMR spectrum for $\left[\mathbf{S}_{\mathbf{2}}-\mathbf{( 2 , 6 - n a p h )}-\mathbf{S}_{\mathbf{2}}\right]$.

- DPV of the dimeric complexes

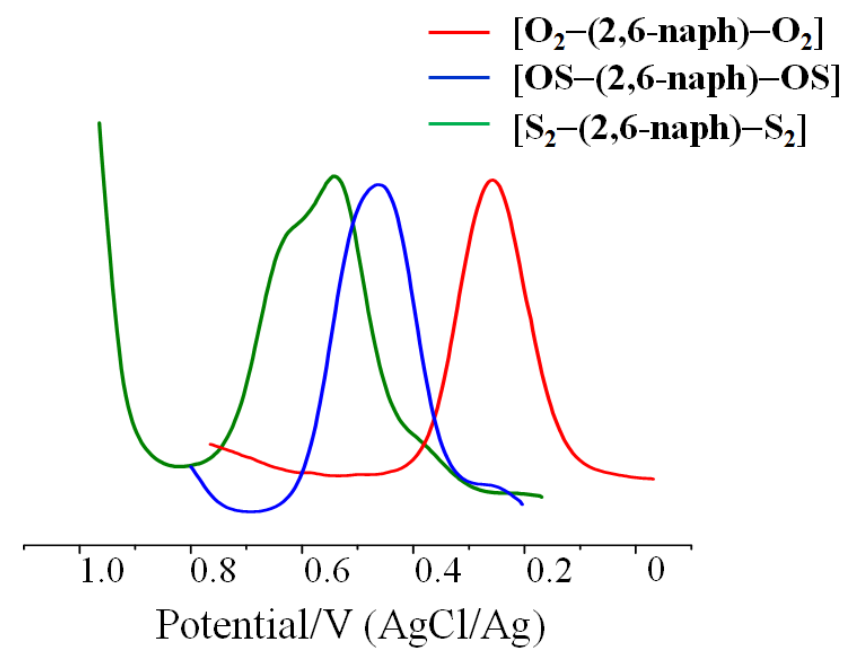

Figure S4. DPV for $\quad\left[\mathrm{O}_{2}-(2,6-n a p h)-O_{2}\right], \quad[$ OS-(2,6-naph)-OS $], \quad$ and $\left[\mathbf{S}_{2}-(\mathbf{2}, \mathbf{6}-\mathbf{n a p h})-\mathbf{S}_{2}\right]$. The amplitude is $0.05 \mathrm{~V}$, the pulse width is $0.2 \mathrm{~s}$ and the sampling interval is $0.5 \mathrm{~s}$. The peak-widths at half height are $135 \mathrm{mV}$ for $\left[\mathbf{O}_{2}-\left(\mathbf{2 , 6}\right.\right.$-naph) $\left.-\mathbf{O}_{2}\right]$ and $169 \mathrm{mV}$ for [OS-(2,6-naph)-OS]. 
- Electronic spectra of the dimeric complexes

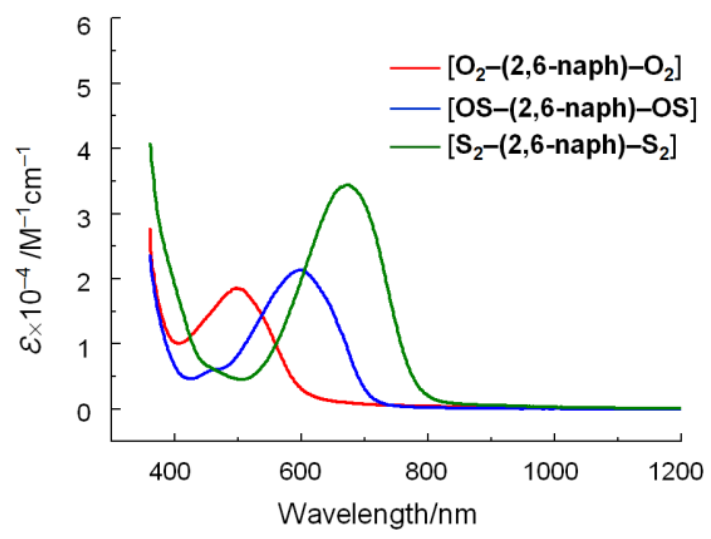

Figure S5. Electronic spectra of the neutral complexes $\left[\mathbf{O}_{2}-(\mathbf{2}, \mathbf{6}-\mathbf{n a p h})-\mathbf{O}_{\mathbf{2}}\right]$, [OS-(2,6-naph)-OS $]$ and $\left[\mathbf{S}_{2}-\mathbf{( 2 , 6 - n a p h )}-\mathbf{S}_{2}\right]$, showing that the band energy $\left(E_{\mathrm{ML}}\right)$ decreases and the intensity increases as the chelating groups of the bridging ligand is stepwise thiolated.

Table S1. Metal to Ligand Charge Transfer Absorptions for the Naphthalene Bridged Complexes in Comparison with the Data for the Phenylene and Biphenylene Bridged Analogues

\begin{tabular}{|c|c|c|c|}
\hline Compd. & $E_{\mathrm{ML}}\left(\mathrm{nm} / \mathrm{cm}^{-1}\right)$ & $\varepsilon_{\mathrm{ML}}\left(\mathrm{M}^{-1} \mathrm{~cm}^{-1}\right)$ & \\
\hline$\left[\mathrm{O}_{2}-(2,6-n a p h)-\mathrm{O}_{2}\right]$ & $499 / 20010$ & 18630 & This work \\
\hline [OS-(2,6-naph)-SO] & $599 / 16700$ & 21330 & \\
\hline$\left[S_{2}-(2,6-n a p h)-S_{2}\right]$ & $673 / 14850$ & 34440 & \\
\hline$\left[\mathbf{O}_{2}-\mathbf{p h}-\mathbf{O}_{2}\right]$ & $485 / 20600$ & 15230 & Ref.12 \\
\hline$[\mathrm{OS}-\mathbf{p h}-\mathrm{OS}]$ & $623 / 16040$ & 25870 & \\
\hline$\left[\mathbf{S}_{2}-\mathbf{p h}-\mathbf{S}_{2}\right]$ & $722 / 13850$ & 39960 & \\
\hline$\left[\mathbf{O}_{2}-\right.$ biph$\left.-\mathbf{O}_{2}\right]$ & $476 / 21012$ & 9272 & Ref.14 \\
\hline [OS-biph-OS] & $577 / 17319$ & 21840 & \\
\hline$\left[\mathbf{S}_{2}-\right.$ biph-S $\left.\mathbf{S}_{2}\right]$ & $639 / 15647$ & 37350 & \\
\hline
\end{tabular}


- EPR spectra of the mixed-valence complexes
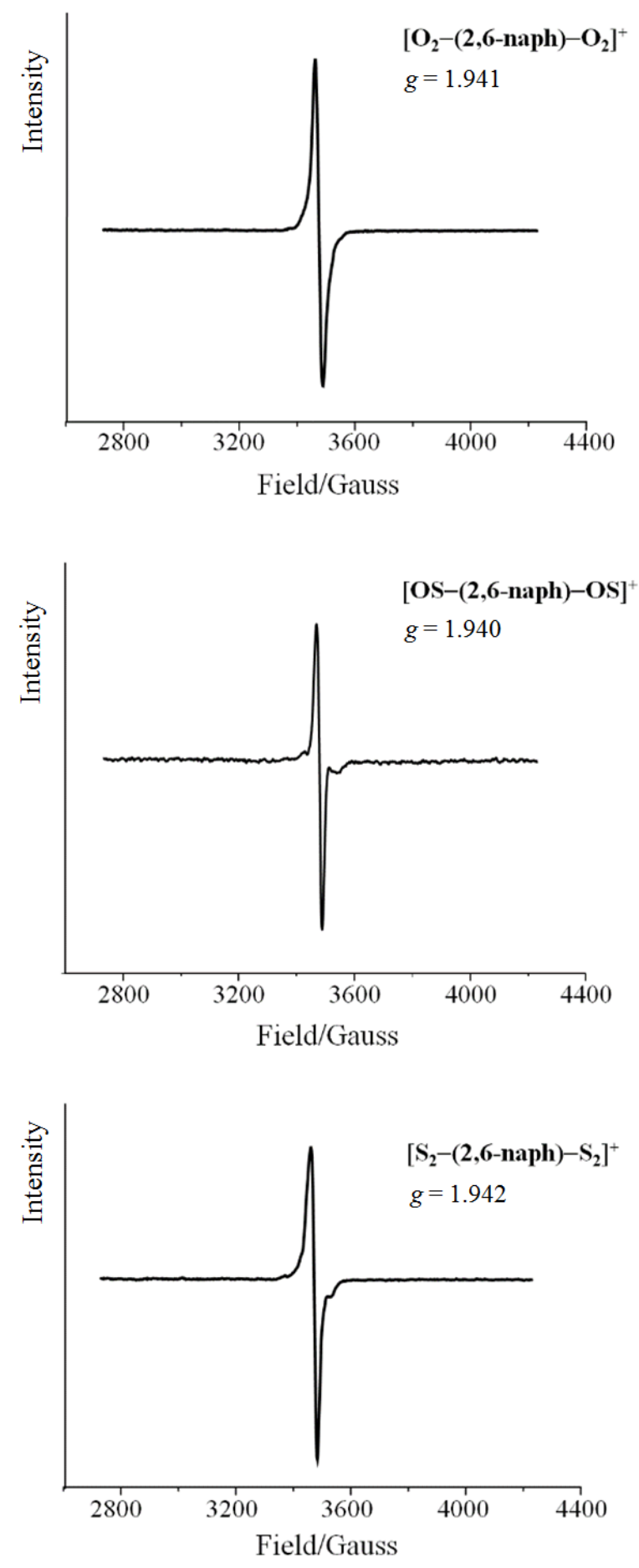

Figure S6. EPR spectra of the radical cations generated by single-electron oxidation of the neutral compounds. Samples were measured in $\mathrm{CH}_{2} \mathrm{Cl}_{2}$ solution at $173 \mathrm{~K}$. 
- Crystallographic data and structural parameters

Table S2. Crystallographic Data for $\left[\mathrm{O}_{2}-(2,6-n a p h)-\mathrm{O}_{2}\right] \cdot 2 \mathrm{CH}_{2} \mathrm{Cl}_{2},[\mathrm{OS}-(2,6-\mathrm{naph})-\mathrm{OS}] \cdot 2 \mathrm{CH}_{2} \mathrm{Cl}_{2},\left[\mathrm{~S}_{2}-\left(2,6-\mathrm{naph}^{2}-\mathrm{S}_{2}\right] \cdot 2 \mathrm{C}_{2} \mathrm{H}_{5} \mathrm{OH}\right.$

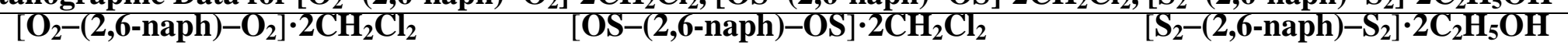

\begin{tabular}{llll}
\hline formula & $\mathrm{C}_{104} \mathrm{H}_{100} \mathrm{Mo}_{4} \mathrm{~N}_{12} \mathrm{O}_{16} \mathrm{Cl}_{4}$ & $\mathrm{C}_{104} \mathrm{H}_{100} \mathrm{Mo}_{4} \mathrm{~N}_{12} \mathrm{O}_{14} \mathrm{~S}_{2} \mathrm{Cl}_{4}$ & $\mathrm{C}_{106} \mathrm{H}_{108} \mathrm{MO}_{4} \mathrm{~N}_{12} \mathrm{O}_{14} \mathrm{~S}_{4}$ \\
fw & 2227.90 & 2260.02 & 2285.14 \\
space group & $P \overline{1}$ & $P_{\overline{1}}$ & $P_{\overline{1}}$ \\
$a(\mathrm{~A})$ & $12.0793(4)$ & $12.4999(3)$ & $9.6079(4)$ \\
$b(\mathrm{~A})$ & $13.5740(5)$ & $14.1504(5)$ & $12.7804(6)$ \\
$c(\mathrm{~A})$ & $15.0188(6)$ & $15.5088(7)$ & $20.3393(8)$ \\
$\alpha(\operatorname{deg})$ & $83.485(3)$ & $105.374(3)$ & $92.208(3)$ \\
$\beta(\mathrm{deg})$ & $84.650(3)$ & $98.551(3)$ & $99.738(3)$ \\
$\gamma(\mathrm{deg})$ & $86.793(3)$ & $96.329(3)$ & $100.073(4)$ \\
$V\left(\mathrm{~A}^{\nu}\right)$ & $2433.4(2)$ & $2583.2(2)$ & $2417.6(2)$ \\
$Z$ & 1 & 1 & 1 \\
$T(\mathrm{~K})$ & 100 & 173 & 173 \\
$d_{\text {calcd }}\left(\mathrm{g} / \mathrm{cm}^{\nu}\right)$ & 1.569 & 1.500 & 1.562 \\
$\mu\left(\mathrm{mm}{ }^{-1}\right)$ & 5.742 & 5.770 & 5.551 \\
$R_{1}{ }^{u}$ & 0.0595 & 0.0583 & 0.0519 \\
$w R_{2}{ }^{\nu}$ & 0.1731 & 0.1710 & 0.1461
\end{tabular}

${ }^{u} R_{1}=\Sigma|| F_{\mathrm{o}}|-| F_{\mathrm{c}}|| \Sigma\left|F_{\mathrm{o}}\right|{ }^{0} w R_{2}=\left[\Sigma\left[w\left(F_{\mathrm{o}}{ }^{2}-F_{\mathrm{c}}{ }^{2}\right)^{2}\right] / \Sigma\left[w\left(F_{\mathrm{o}}{ }^{2}\right)^{2}\right]\right]^{1 / 2}$ 
Table S3. Selected Bond Distances $(\AA)$ of $\left[\mathrm{O}_{2}-(2,6-\mathrm{naph})-\mathrm{O}_{2}\right] \cdot 2 \mathrm{CH}_{2} \mathrm{Cl}_{2},[\mathrm{OS}-(2,6-\mathrm{naph})-\mathrm{OS}] \cdot 2 \mathrm{CH}_{2} \mathrm{Cl}_{2},\left[\mathrm{~S}_{2}-(2,6-\mathrm{naph})-\mathrm{S}_{2}\right] \cdot 2 \mathrm{C}_{2} \mathrm{H}_{5} \mathrm{OH}$ $\left[\mathrm{O}_{2}-(2,6-\mathrm{naph})-\mathrm{O}_{2}\right] \cdot 2 \mathrm{CH}_{2} \mathrm{Cl}_{2} \quad[\mathrm{OS}-(2,6-\mathrm{naph})-\mathrm{OS}] \cdot 2 \mathrm{CH}_{2} \mathrm{Cl}_{2} \quad\left[\mathrm{~S}_{2}-(2,6-\mathrm{naph})-\mathrm{S}_{2}\right] \cdot 2 \mathrm{C}_{2} \mathrm{H}_{5} \mathrm{OH}$

\begin{tabular}{|c|c|c|c|}
\hline $\operatorname{Mo}(1)-\operatorname{Mo}(2)$ & 2.089 & 2.099 & 2.104 \\
\hline $\mathrm{Mo}(1)-\mathrm{O}(7)$ & 2.114 & & \\
\hline $\mathrm{Mo}(2)-\mathrm{O}(8)$ & 2.158 & 2.095 & \\
\hline $\operatorname{Mo}(1)-S(1)$ & & 2.461 & 2.399 \\
\hline $\operatorname{Mo}(2)-S(2)$ & & & 2.451 \\
\hline$C(4)-C(5)$ & 1.490 & 1.472 & 1.469 \\
\hline $\mathrm{C}(4)-\mathrm{S}(1)$ & & 1.704 & 1.664 \\
\hline$C(4)-S(2)$ & & & 1.680 \\
\hline $\mathrm{C}(4)-\mathrm{O}(7)$ & 1.270 & & \\
\hline $\mathrm{C}(4)-\mathrm{O}(8)$ & 1.278 & 1.251 & \\
\hline $\mathrm{Mo}_{2} \ldots \mathrm{Mo}_{2}$ & 13.332 & 13.696 & 14.280 \\
\hline$\phi(1)$ & 7.64 & 4.57 & 23.18 \\
\hline$\phi(2)$ & 5.64 & 4.58 & 23.75 \\
\hline
\end{tabular}

${ }^{a} \phi(1)$ refers to $\mathrm{O}(7) / \mathrm{S}(1)-\mathrm{C}(4)-\mathrm{C}(5)-\mathrm{C}(8)$, and $\phi(2)$ to $\mathrm{O}(8) / \mathrm{S}(2)-\mathrm{C}(4)-\mathrm{C}(5)-\mathrm{C}(9)$ 
- DFT Calculation Data

Table S4. Calculated Bond Distances and Energy Levels of the Selected Frontier Molecular Orbitals

\begin{tabular}{|c|c|c|c|c|c|c|c|c|}
\hline \multirow[b]{2}{*}{ model } & \multicolumn{4}{|c|}{ bond distance $(\AA)$} & \multirow{2}{*}{$\begin{array}{l}\text { HOMO-LUMO } \\
\text { energy gap }(\mathrm{eV})\end{array}$} & \multirow{2}{*}{$\begin{array}{c}\text { HOMO-HOMO-1 } \\
\text { energy gap }(\mathrm{eV})\end{array}$} & \multicolumn{2}{|c|}{$\operatorname{MLCT}\left(\mathrm{cm}^{-1}\right)$} \\
\hline & Mo-Mo & $\mathrm{Mo}_{2} \ldots \mathrm{Mo}_{2}$ & Mo-O & Mo-S & & & $\overline{\operatorname{expt}}$ & calcd $^{a}$ \\
\hline$\left[\mathrm{O}_{2}-(2,6-n a p h)-\mathrm{O}_{2}\right]$ & 2.129 & 13.482 & $2.145 \quad 2.154$ & & 2.469 & 0.099 & 20010 & 19917 \\
\hline [OS-(2,6-naph)-SO] & 2.136 & 13.884 & 2.112 & 2.528 & 2.278 & 0.153 & 16700 & 18376 \\
\hline$\left[S_{2}-(2,6-n a p h)-S_{2}\right]$ & 2.139 & 14.563 & & $2.488 \quad 2.495$ & 2.190 & 0.196 & 14850 & 17666 \\
\hline$\left[\mathbf{O}_{2}-\mathrm{ph}-\mathrm{O}_{2}\right]$ & 2.116 & 11.320 & 2.144 & & 2.26 & 0.13 & 20600 & 18230 \\
\hline$[\mathrm{OS}-\mathbf{p h}-\mathrm{OS}]$ & 2.124 & 11.769 & 2.104 & 2.498 & 1.90 & 0.22 & 16040 & 15330 \\
\hline$\left[\mathbf{S}_{2}-\mathbf{p h}-\mathbf{S}_{2}\right]$ & 2.127 & 12.383 & & 2.466 & 1.81 & 0.27 & 13850 & 14600 \\
\hline
\end{tabular}

${ }^{a}$ The values $\left(\mathrm{cm}^{-1}\right)$ converted from the HOMO-LUMO energy gap $(\mathrm{eV})$ 
Data S1. Calculated Geometrical Data for $\left[\mathrm{O}_{2}-(2,6-n a p h)-\mathbf{O}_{2}\right]$

Mo

Mo

$\mathrm{O}$

$\mathrm{O}$

$\mathrm{N}$

$\mathrm{N}$

$\mathrm{N}$

$\mathrm{N}$

$\mathrm{N}$

$\mathrm{N}$

$\mathrm{C}$

$\mathrm{H}$

$\mathrm{C}$

$\mathrm{H}$

C

$\mathrm{H}$

C

C

$\mathrm{H}$

C

C

$\mathrm{H}$

C

C

$\mathrm{H}$

Mo

Mo

$\mathrm{O}$

$\mathrm{O}$

$\mathrm{N}$

$\mathrm{N}$

$\mathrm{N}$

$\mathrm{N}$

$\mathrm{N}$

$\mathrm{N}$

C

$\mathrm{H}$

C

$\mathrm{H}$

C

$\mathrm{H}$

C

C
$-6.85159000$

$-6.65585300$

$-4.71527700$

$-4.51119400$

$-8.77678900$

$-6.55268400$

$-8.98829400$

$-6.76652200$

$-6.55721100$

$-6.76828000$

$-9.54744300$

$-10.63626200$

$-6.59418400$

$-6.48063500$

$-6.59992300$

$-6.49074300$

$-2.51955900$

$-0.56952700$

$-0.12822900$

$-0.29649500$

$-1.93201400$

$-2.58494800$

$-3.99883100$

$-1.70474400$

$-2.15739200$

6.82827800

6.67914300

4.69240200

4.52693100

8.80620800

6.57481000

8.96775400

6.73850600

6.58872600

6.75369500

9.55292400

10.64368000

6.59159900

6.47889500

6.61142200

6.50743300

2.51368800

0.56237700
0.07056400

$-0.07175900$

0.08367000

$-0.06180900$

$-0.09196000$

$-2.22383400$

0.06163000

$-2.07103100$

2.06978300

2.22486200

$-0.01977100$

$-0.02752200$

$-2.80843100$

$-3.89337500$

2.80947200

3.89617600

0.02257800

0.12661800

0.19942400

$-0.05024100$

0.11684400

0.18142500

0.01388100

$-0.05870900$

$-0.12959000$

$-0.02511400$

0.02899100

$-0.03901300$

0.02100000

0.04129200

2.18249900

$-0.01661600$

2.12390000

$-2.12033600$

$-2.18038100$

0.01559100

0.02094800

2.81588100

3.90350500

$-2.81377100$

$-3.90265500$

$-0.02545000$

$-0.13049500$
1.03539900

$-1.07857200$

1.28110000

$-0.93765400$

$-1.35544000$

$-1.00664400$

0.91997400

1.26942100

$-1.29652200$

0.97948800

$-0.27921900$

$-0.38013700$

0.18200600

0.26558400

$-0.19789900$

$-0.26222400$

0.37271100

1.81142700

2.80136600

$-0.61856200$

1.66404400

2.52561600

0.22809300

$-0.73982300$

$-1.72251800$

$-1.05124500$

1.07141000

$-1.24930500$

0.97678500

1.29714600

1.09240100

$-0.98678600$

$-1.19170000$

1.20159700

$-1.08233800$

0.20219600

0.27973100

$-0.07137900$

$-0.10752100$

0.07280000

0.09437700

$-0.31161700$

$-1.74809200$ 
$\mathrm{H}$

C

C

$\mathrm{H}$

C

C

$\mathrm{H}$

$\mathrm{H}$

$\mathrm{H}$

$\mathrm{H}$

$\mathrm{H}$

$\mathrm{H}$

$\mathrm{H}$

$\mathrm{H}$

$\mathrm{H}$

$\mathrm{H}$

$\mathrm{H}$

$\mathrm{H}$

$\mathrm{H}$
0.11917200

0.29193800

1.92417500

2.57627900

3.99437600

1.70134800

2.15583400

$-6.76999400$

$-6.40535200$

$-6.39661800$

$-6.76611700$

$-9.29073400$

$-9.65460300$

6.74219400

6.45540900

6.43714300

6.72121300

9.61695700

9.33925700
$-0.20289000$

0.04353700

$-0.11661600$

$-0.17643400$

$-0.01344100$

0.05109200

0.11965700

2.86956800

2.60224300

$-2.86600800$

$-2.60352900$

$-0.15235000$

0.11340700

$-2.79206800$

$-2.68775300$

2.79200500

2.69172900

$-0.03547600$

0.06606700
$-2.73718800$

0.68351700

$-1.60258000$

$-2.46486300$

$-0.18163900$

0.80327100

1.78547700

1.75987600

$-2.14396700$

$-1.77344000$

2.13029500

$-2.22550200$

1.68030900

$-1.88875300$

2.02922000

1.88877200

$-2.02950700$

$-1.76321500$

2.15741200

Data S2. Calculated Geometrical Data for [OS-(2,6-naph)-OS]

$\begin{array}{lrrr}\text { Mo } & 7.28190500 & 0.95193800 & -0.12176300 \\ \text { Mo } & 6.60154000 & -1.05618500 & 0.13328400 \\ \text { S } & 4.93860700 & 1.89300400 & -0.23963600 \\ \text { O } & 4.52355900 & -0.67948100 & 0.08191500 \\ \mathrm{~N} & 8.61170900 & -1.78886300 & 0.23268000 \\ \mathrm{~N} & 6.52762200 & -1.38487100 & -1.99265200 \\ \mathrm{~N} & 9.34887200 & 0.36452300 & -0.04248500 \\ \mathrm{~N} & 7.28797200 & 0.76181100 & -2.27040500 \\ \mathrm{~N} & 6.51723000 & -0.84403800 & 2.27372100 \\ \mathrm{~N} & 7.27552400 & 1.30470700 & 2.00624600 \\ \mathrm{C} & 9.61450900 & -0.92508700 & 0.12319100 \\ \mathrm{H} & 10.65077900 & -1.27561200 & 0.16958100 \\ \mathrm{C} & 6.87350900 & -0.38359200 & -2.79557800 \\ \mathrm{H} & 6.80769700 & -0.50102700 & -3.88218900 \\ \mathrm{C} & 6.85957500 & 0.32725200 & 2.80073400 \\ \mathrm{H} & 6.78943400 & 0.48628700 & 3.88177500 \\ \mathrm{C} & 2.49503800 & 0.53263000 & -0.05952600 \\ \mathrm{C} & 0.43016100 & 1.81468600 & -0.18041100 \\ \mathrm{H} & -0.08659600 & 2.76709000 & -0.27264800 \\ \mathrm{C} & 0.34422700 & -0.62560500 & 0.06078100 \\ \mathrm{C} & 1.80203400 & 1.77385800 & -0.18090200 \\ \mathrm{H} & 2.37773700 & 2.68831800 & -0.27312900 \\ \mathrm{C} & 3.97597800 & 0.45806300 & -0.05563400\end{array}$




\begin{tabular}{|c|c|c|c|}
\hline $\mathrm{C}$ & 1.75781800 & -0.63951600 & 0.05880900 \\
\hline $\mathrm{H}$ & 2.28110600 & -1.58602300 & 0.15121500 \\
\hline Mo & -7.28190200 & -0.95192900 & 0.12187200 \\
\hline Mo & -6.60154500 & 1.05617300 & -0.13336400 \\
\hline S & -4.93859900 & -1.89298700 & 0.23971500 \\
\hline $\mathrm{O}$ & -4.52356300 & 0.67947400 & -0.08203600 \\
\hline $\mathrm{N}$ & -8.61171900 & 1.78884300 & -0.23274300 \\
\hline $\mathrm{N}$ & -6.52754600 & 1.38503500 & 1.99254100 \\
\hline $\mathrm{N}$ & -9.34887100 & -0.36451900 & 0.04263900 \\
\hline $\mathrm{N}$ & -7.28787400 & -0.76162900 & 2.27050100 \\
\hline $\mathrm{N}$ & -6.51731500 & 0.84385000 & -2.27378700 \\
\hline $\mathrm{N}$ & -7.27561300 & -1.30486900 & -2.00610800 \\
\hline $\mathrm{C}$ & -9.61451400 & 0.92507800 & -0.12313600 \\
\hline $\mathrm{H}$ & -10.65078600 & 1.27560000 & -0.16951200 \\
\hline $\mathrm{C}$ & -6.87339400 & 0.38381900 & 2.79556300 \\
\hline $\mathrm{H}$ & -6.80753800 & 0.50134300 & 3.88216100 \\
\hline $\mathrm{C}$ & -6.85968800 & -0.32748100 & -2.80069100 \\
\hline $\mathrm{H}$ & -6.78959000 & -0.48660400 & -3.88172200 \\
\hline $\mathrm{C}$ & -2.49503600 & -0.53262600 & 0.05944000 \\
\hline $\mathrm{C}$ & -0.43015900 & -1.81468200 & 0.18032400 \\
\hline $\mathrm{H}$ & 0.08659800 & -2.76708500 & 0.27256700 \\
\hline $\mathrm{C}$ & -0.34422500 & 0.62560800 & -0.06088200 \\
\hline $\mathrm{C}$ & -1.80203100 & -1.77385300 & 0.18083100 \\
\hline $\mathrm{H}$ & -2.37773400 & -2.68831100 & 0.27307400 \\
\hline $\mathrm{C}$ & -3.97597700 & -0.45806100 & 0.05556700 \\
\hline $\mathrm{C}$ & -1.75781600 & 0.63951900 & -0.05891300 \\
\hline $\mathrm{H}$ & -2.28110500 & 1.58602600 & -0.15131800 \\
\hline $\mathrm{H}$ & 8.90767600 & -2.75188300 & 0.35618800 \\
\hline $\mathrm{H}$ & 10.17403300 & 0.95098300 & -0.11679100 \\
\hline $\mathrm{H}$ & 6.20634100 & -2.21233700 & -2.48544600 \\
\hline $\mathrm{H}$ & 7.49985000 & 1.47618100 & -2.95980700 \\
\hline $\mathrm{H}$ & -6.19344500 & 1.52150800 & -2.95688200 \\
\hline $\mathrm{H}$ & -7.48394000 & -2.16974900 & -2.49511900 \\
\hline $\mathrm{H}$ & -7.49972000 & -1.47594300 & 2.95997000 \\
\hline $\mathrm{H}$ & -6.20625000 & 2.21254200 & 2.48525400 \\
\hline $\mathrm{H}$ & 6.19333600 & -1.52175400 & 2.95674800 \\
\hline $\mathrm{H}$ & 7.48382700 & 2.16954900 & 2.49533500 \\
\hline $\mathrm{H}$ & -10.17402900 & -0.95097200 & 0.11703000 \\
\hline $\mathrm{H}$ & -8.90769000 & 2.75185300 & -0.35632000 \\
\hline
\end{tabular}

Data S3. Calculated Geometrical Data for [ $\left.\mathbf{S}_{\mathbf{2}}-\mathbf{( 2 , 6 - n a p h )}-\mathbf{S}_{\mathbf{2}}\right]$

$\begin{array}{llrc}\text { Mo } & -7.35607000 & 0.72081900 & 0.75290800 \\ \text { Mo } & -7.20666700 & -0.77477400 & -0.76863400 \\ \text { S } & -4.94388800 & 1.20125700 & 1.13018700\end{array}$




$\begin{array}{ccc}-4.72733900 & -0.88141400 & -1.02248600 \\ -9.35470600 & -0.98323800 & -0.88143200 \\ -7.15201600 & -2.36341100 & 0.69326800 \\ -9.51497900 & 0.61750700 & 0.74788900 \\ -7.30492000 & -0.76162500 & 2.32095200 \\ -7.29354100 & 0.70764700 & -2.33742500 \\ -7.44540900 & 2.30809500 & -0.70869800 \\ -10.09550600 & -0.23080900 & -0.08459700 \\ -11.18659100 & -0.31006200 & -0.11429900 \\ -7.20030200 & -2.03458800 & 1.97437700 \\ -7.13991900 & -2.81010900 & 2.74319600 \\ -7.38329600 & 1.98118800 & -1.98954600 \\ -7.39211200 & 2.76077500 & -2.75655900 \\ -2.53193700 & 0.34168000 & 0.12217500 \\ -0.55007200 & 1.66382500 & 0.64387600 \\ -0.10371300 & 2.58892500 & 0.99721900 \\ -0.30327100 & -0.60194300 & -0.23769500 \\ -1.91226300 & 1.53956100 & 0.59439900 \\ -2.54143500 & 2.36440900 & 0.90428800 \\ -4.00428400 & 0.22413600 & 0.07539400 \\ -1.70994400 & -0.70140200 & -0.28389200 \\ -2.15582200 & -1.62482600 & -0.63579500 \\ 7.35611000 & -0.72079500 & -0.75286200 \\ 7.20664300 & 0.77458500 & 0.76888500 \\ 4.94393600 & -1.20090000 & -1.13060800 \\ 4.72726300 & 0.88152800 & 1.02227800 \\ 9.35469000 & 0.98271000 & 0.88209200 \\ 7.15249500 & 2.36345200 & -0.69278500 \\ 9.51503600 & -0.61779100 & -0.74746200 \\ 7.30543600 & 0.76189800 & -2.32069500 \\ 7.29302000 & -0.70809900 & 2.33745300 \\ 7.44498000 & -2.30831400 & 0.70850900 \\ 10.09552800 & 0.23032100 & 0.08525600 \\ 11.18661800 & 0.30943900 & 0.11515100 \\ 7.20095100 & 2.03482300 & -1.97393700 \\ 7.14081400 & 2.81047400 & -2.74264400 \\ 7.38267400 & -1.98159900 & 1.98939600 \\ 7.39124400 & -2.76130400 & 2.75629000 \\ 2.53190900 & -0.34132900 & -0.12275100 \\ 0.55004500 & -1.66345900 & -0.64451300 \\ 0.10368900 & -2.588566000 & -0.99784000 \\ 0.30323300 & 0.60230400 & 0.23706400 \\ 1.91223700 & -1.53919600 & -0.59501800 \\ 2.54141500 & -2.36405500 & -0.90486400\end{array}$




$\begin{array}{lrrr}\mathrm{C} & 4.00425800 & -0.22382700 & -0.07583400 \\ \mathrm{C} & 1.70990300 & 0.70175300 & 0.28329700 \\ \mathrm{H} & 2.15577000 & 1.62516400 & 0.63524800 \\ \mathrm{H} & -7.48271400 & 3.30515600 & -0.53942000 \\ \mathrm{H} & -7.22093500 & 0.55767100 & -3.33568700 \\ \mathrm{H} & -7.04596400 & -3.35599200 & 0.52646100 \\ \mathrm{H} & -7.30341900 & -0.60606800 & 3.32099200 \\ \mathrm{H} & -9.88572200 & -1.60064700 & -1.48215800 \\ \mathrm{H} & -10.16171000 & 1.14912100 & 1.31629700 \\ \mathrm{H} & 7.48217800 & -3.30535300 & 0.53908000 \\ \mathrm{H} & 7.22024100 & -0.55827000 & 3.33572400 \\ \mathrm{H} & 7.04656600 & 3.35602300 & -0.52584000 \\ \mathrm{H} & 7.30408500 & 0.60650000 & -3.32076000 \\ \mathrm{H} & 10.16179600 & -1.14938800 & -1.31585300 \\ \mathrm{H} & 9.88568300 & 1.59995200 & 1.48300900\end{array}$

